\title{
Articles of Significant Interest Selected from This Issue by the Editors
}

\section{Adaptation of Influenza H5N1 Virus to Humans}

A lysine residue at position 627 in the influenza virus PB2 protein is associated with transmission of highly pathogenic H5N1 viruses between ferrets. Long et al. (p. 9983-9996) examine the effect of this mutation on virus replication in avian species. An historic pre-1997 H5N1 virus does not tolerate the PB2 Lys627 mutation, whereas a modern H5N1 virus replicates well. This knowledge underpins our ability to calculate the risk of future pandemics by influenza viruses.

\section{Redefining the Roles of E1A Targets in Cellular Transformation}

The C-terminal region of adenovirus E1A is required for transformation in cooperation with E1B. However, the E1A C terminus paradoxically suppresses transformation in cooperation with activated Ras. Cohen et al. (p. 10348-10355) report that the interaction of the C terminus of E1A with importin $\alpha 3$ /Qip1, DYRK1A, HAN11, and CtBP influences transformation with E1B-55K. Moreover, interactions of E1A with DYRK1A and HAN11 but not CtBP suppress transformation by activated Ras. These data revise the current model of how E1A mediates transformation and suggest a key role for DYRK1A and HAN11 in this process.

\section{Sequential Steps in ICP0 and ND10 Interactions}

Interactions between host cell nuclear structure ND10 and herpes simplex virus 1 (HSV-1) ICP0 is part of the initial host-virus contest that determines the outcome of HSV-1 infection. Gu et al. (p. 10244-10254) show that the ND10-ICP0 interaction is dynamic and includes sequential steps of adhesion, fusion, and retention, each requiring specific domains of ICP0. This work deciphers the mechanism of ND10-ICP0 interactions and identifies a new drug target for treatment of herpesvirus infections.

\section{Direct Binding of Ifit1 to Viral 5'-Capped 2'-0 Unmethylated RNA}

Members of the interferon-inducible protein with tetratricopeptide (Ifit) family inhibit viral replication by recognizing several types of viral RNA. Kimura et al. (p. 9997-10003) use a 2'-O methyltransferase mutant of Japanese encephalitis virus to show that Ifit1 directly interacts with $5^{\prime}$ capped viral mRNA without $2^{\prime}-\mathrm{O}$ methylation, thereby inhibiting its translation. These results reveal a key mechanism for Ifit1-mediated antiviral responses.

\section{HIV Populations In Vivo Are Driven by Selection and Genetic Drift}

Many of the challenges limiting the development of an effective HIV vaccine or the durability of antiretroviral therapy can be traced directly to the rapid and error-prone virus replication program. Maldarelli et al. (p. 10313-10323) quantified the replicating population size, diversity, and divergence of HIV populations in vivo and found that replicating HIV populations are large, diverse, and slow to change. These data indicate that both selection and drift are responsible for emergence of new viral variants. 